\title{
Article
}

\section{Mapping Inversion Domain Boundaries along Single GaN Wires with Bragg Coherent X-Ray Imaging}

Ni Li, Stephane Labat, Steven J. Leake, Maxime Dupraz, Jérôme Carnis, Thomas W. Cornelius, Guillaume Beutier, Marc Verdier, Vincent Favre-Nicolin,

Tobias U. Schulli, Olivier Thomas, Joel Eymery, and Marie-Ingrid Richard

ACS Nano, Just Accepted Manuscript • DOI: 10.1021/acsnano.0c03775 • Publication Date (Web): 05 Aug 2020

Downloaded from pubs.acs.org on August 6, 2020

\section{Just Accepted}

"Just Accepted" manuscripts have been peer-reviewed and accepted for publication. They are posted online prior to technical editing, formatting for publication and author proofing. The American Chemical Society provides "Just Accepted" as a service to the research community to expedite the dissemination of scientific material as soon as possible after acceptance. "Just Accepted" manuscripts appear in full in PDF format accompanied by an HTML abstract. "Just Accepted" manuscripts have been fully peer reviewed, but should not be considered the official version of record. They are citable by the Digital Object Identifier (DOI®). "Just Accepted" is an optional service offered to authors. Therefore, the "Just Accepted" Web site may not include all articles that will be published in the journal. After a manuscript is technically edited and formatted, it will be removed from the "Just Accepted" Web site and published as an ASAP article. Note that technical editing may introduce minor changes to the manuscript text and/or graphics which could affect content, and all legal disclaimers and ethical guidelines that apply to the journal pertain. ACS cannot be held responsible for errors or consequences arising from the use of information contained in these "Just Accepted" manuscripts. 


\title{
Mapping Inversion Domain Boundaries along Single GaN Wires with Bragg Coherent X-Ray Imaging
}

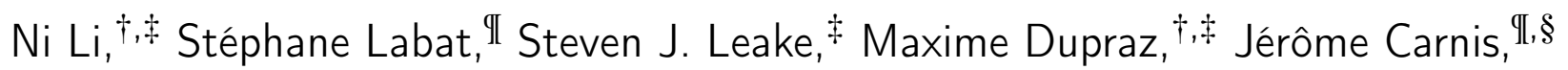 \\ Thomas W. Cornelius, "I Guillaume Beutier, "I Marc Verdier, "Vincent \\ Favre-Nicolin, ${ }^{\ddagger}$ Tobias U. Schülli, ${ }^{\ddagger}$ Olivier Thomas, ${ }^{, I}$ Joël Eymery, ${ }^{\dagger}$ and \\ Marie-Ingrid Richard*,,$+ \neq$ \\ $\dagger$ Univ. Grenoble Alpes, CEA Grenoble, IRIG, MEM, NRS, 17 rue des Martyrs 38000 Grenoble, \\ France \\ $\ddagger E S R F$ - The European Synchrotron, 71 Avenue des Martyrs, Grenoble 38000, France \\ IIAix Marseille Université, CNRS, Université de Toulon, IM2NP UMR 7334, 13397 Marseille, \\ France \\ §Deutsches Elektronen-Synchrotron (DESY), Notkestraße 85, 22607 Hamburg \\ ||Univ. Grenoble Alpes, CNRS, Grenoble INP, SIMaP, 38000 Grenoble, France \\ E-mail: mrichard@esrf.fr
}

\begin{abstract}
Gallium nitride $(\mathrm{GaN})$ is of technological importance for a wide variety of optoelectronic applications. Defects in GaN, like inversion domain boundaries (IDBs), significantly affect the electrical and optical properties of the material. We report, here, on the structural configurations of planar
\end{abstract}


inversion domain boundaries inside $n$-doped GaN wires measured by Bragg coherent $\mathrm{x}$-ray diffraction imaging. Different complex domain configurations are revealed along the wires with a 9-nm in-plane spatial resolution. We demonstrate that the IDBs change their direction of propagation along the wires, promoting Ga-terminated domains and stabilising into $\{1 \overline{1} 00\}$ i.e. $m$-planes. The atomic phase shift between the $\mathrm{Ga}$ - and $\mathrm{N}$ - terminated domains has been extracted using phase retrieval algorithms, revealing an evolution of the out-of-plane displacement ( $\sim 5 \mathrm{pm}$, at maximum) between inversion domains along the wires. This work provides an accurate inner view of planar defects inside small crystals.

\section{Keywords}

GaN wires, inversion domain boundary, coherent x-ray Bragg imaging, displacement field, polarity.

Group-III nitrides play an important role as electronic high-power devices, ${ }^{1}$ photocatalytic ${ }^{2}$ and optoelectronic materials, ${ }^{3}$ thank to their wide bandgaps enabling the realisation of efficient blue-UV light diodes and lasers. Among all the group-III nitride materials, GaN heterostructures have a particular importance owing to their outstanding luminescence efficiency even with a high density of extended defects. ${ }^{4}$ Due to the lack of suitable substrate with a similar lattice parameter, GaN typically exhibits a high defect density, when grown epitaxially. Of many possible defects, inversion domain boundaries (IDBs) correspond to interfaces between two opposite orientation domains of the hexagonal lattice. These domains are related by an inversion symmetry operation across the boundary. Here, we will consider domains along the $c$-orientation. By convention, in the $+c$ domain, the Ga-N bonds are oriented along the [0001] direction, also called the Gapolar orientation. ${ }^{5}$ This polarity mixing has an important role on the growth mechanisms, for example, on the formation of non-polar facets in GaN crystals ${ }^{6,7}$ and on the dopant incorporation. ${ }^{8}$ It can also directly drive the optical and electrical properties of $\mathrm{GaN}, e . g$., for the realisation of 
two-dimensional (2D) electron gas with $\mathrm{AlGaN} / \mathrm{GaN}$ heterostructures in high-mobility and highfrequency transistors ${ }^{9}$ or with the deposition of InGaN/GaN multiple quantum wells. ${ }^{10} \mathrm{GaN}$ wirebased structures have been proposed to increase performance of optoelectronic devices. The large aspect ratio and the small diameter of the wires allow filtering threading dislocations along the wire by bending their dislocation line to the sidewall surfaces. ${ }^{11}$ The resulting very good crystalline quality also provides interesting mechanical properties that has been used in flexible LEDs ${ }^{12}$ and flexible piezoelectric devices. ${ }^{13}$

The study of the inner structure of GaN wire at the nanometer scale is crucial to gain an understanding on their growth mechanisms and properties. Transmission electron microscopy (TEM) is the usual technique to study extended defects, and recent direct observation of IDBs in GaN has been demonstrated with a sub-ångstrom resolution. ${ }^{14}$ However, thinning of samples is always needed to limit electron absorption by the materials, so that only a slice (with rather limited size) can be observed at the same time. Bragg coherent x-ray diffraction imaging (BCDI) ${ }^{15-18}$ was chosen in this work to image the IDB defect of GaN wires epitaxially grown on sapphire without any destructive thinning. BCDI and other related coherent techniques have already been used to image wires ${ }^{19-21}$ and IDB defect. ${ }^{16}$ Here, the measurements provide clues of the structure and propagation of IDBs along the full length of the wires as well as their related displacement and strain fields. This study brings original structural measurement of the evolution of an isolated pure phase shift object (here, inversion domain boundaries) in contrast to other studies focusing mainly on strain gradients, interfacial strains ${ }^{21}$ or assembly of defects. ${ }^{19,20}$

\section{Results/Discussion}

The samples consist of self-catalysed GaN wires grown on $c$-plane sapphire substrates by metal organic vapor phase epitaxy (MOVPE) using silane addition and low V/III ratio (see the Methods and Experimental Section for growth details). ${ }^{6,8,16}$ A low density $\left(\sim 10^{6} \mathrm{~cm}^{-2}\right)$ of wires with a length ranging from 3 to $5 \mu \mathrm{m}$ and an average diameter of $600 \mathrm{~nm}$ were grown, as illustrated by the 


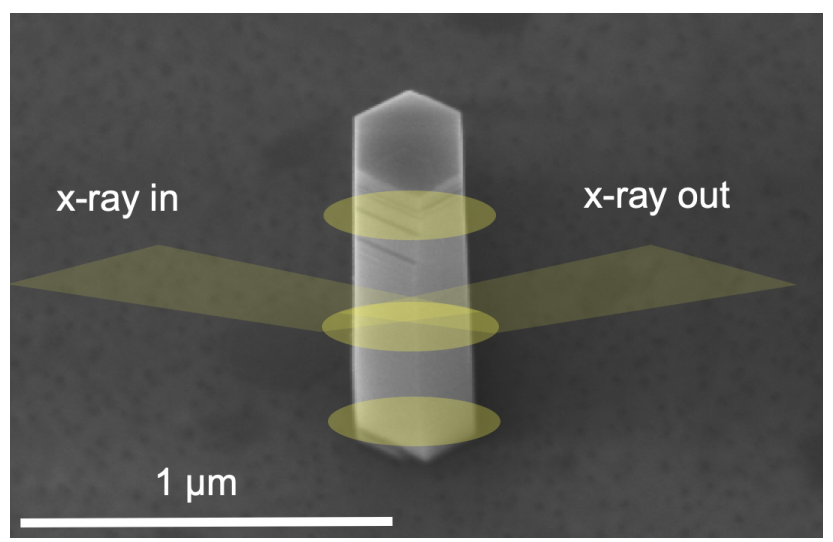

Figure 1: Scanning electron microscope (SEM) image of a GaN wire. The position of the x-ray beam for the different measurements along the wires is indicated by yellow disks.

scanning electron microscopy (SEM) image of Figure 1. In these specific MOVPE growth conditions, ${ }^{6,8,12,13}$ the wires are in majority $\mathrm{N}$-polar, i.e. they grow along the $\bar{c}$-axis $([000 \overline{1}]$ direction) and have six lateral $\{1 \overline{1} 00\} m$-plane facets. This work focuses on three different GaN wires, noted $A, B$ and $C$ hereafter. They were selected at the wafer border to increase the probability to observe IDB defects. At this position, wires are shorter than at the center of the wafer and may have a quite large length distribution coming from different captured volumes of gas phase precursors. Wire $A$ was grown for $150 \mathrm{~s}$ under a silane flow of $\sim 200 \mathrm{nmol} / \mathrm{min}$ (see Supporting Information $\mathrm{S} 1)$. Wires $B$ and $C$ are from the same sample, whose growth was performed for $100 \mathrm{~s}$ under a silane flow of $\sim 200 \mathrm{nmol} / \mathrm{min}$ and continued during $300 \mathrm{~s}$ without silane injection. This second step results in a smaller growth rate and consumes the residual silane in the reactor. The length of wires $A, B$ and $C$ is $5 \mu \mathrm{m}, 3.6 \mu \mathrm{m}$ and $4.3 \mu \mathrm{m}$, respectively (see Figure $\mathrm{S} 1$ ). BCDI was performed using a nano-focused $\mathrm{x}$-ray beam with a size of 430 (vertically) $\times 700$ (horizontally) $\mathrm{nm}^{2}$ at beamline ID01 of the European Synchrotron Facility (ESRF) in Grenoble (France) (see more details in Refs. ${ }^{16,22}$ and in the Methods and Experimental Section).

Three-dimensional diffraction patterns were collected at the $004 \mathrm{GaN}$ Bragg reflection and at three different heights along the wire axis, while illuminating a $\sim 400 \mathrm{~nm}$-thick slice corresponding to the vertical beam size (see Figure 1). The distance between adjacent diffraction patterns are $1.5 \mu \mathrm{m}$ for wire $A$ and $1 \mu \mathrm{m}$ for wires $B$ and $C$. To measure the full three-dimensional diffraction 

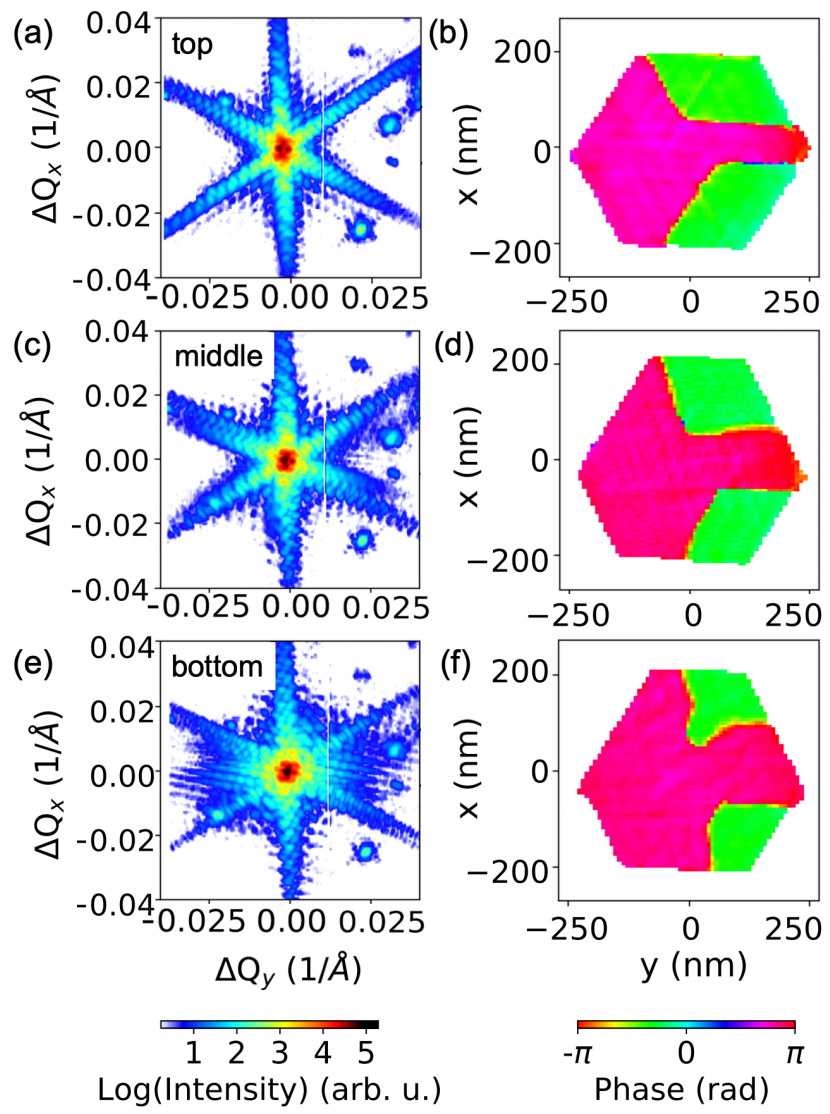

Figure 2: Coherent x-ray diffraction measurements of the $004 \mathrm{GaN}$ Bragg peak (left) and their associated real space reconstruction of the phase (right) at the top (a-b), center (c-d) and bottom (e-f) of the $5 \mu \mathrm{m}$ long wire $A$.

patterns, the wires were rotated through $1.28^{\circ}$, in steps of $0.005^{\circ}$. In all 3D diffraction patterns, the measured intensity is concentrated in an $(h, k)$-plane perpendicular to the $l$-axis. 2D intensity maps are then extracted from the 3D data sets to reconstruct a 2D image of the sample (see Figures 2, 3 and 4 - right side ( $b, d$ and $f)$ ). The images correspond to the projection of the measured 3D portion along the $c$-axis of the wire onto 2D.

Figures 2, 3 and 4 (a, c and e) display coherent $\mathrm{x}$-ray diffraction measurements as a function of the in-plane coordinates of the scattering vector $\vec{Q}\left(Q_{x}\right.$ and $\left.Q_{y}\right)$ at different heights along the wires. Interestingly, the diffraction patterns are different from one wire to another, but they show some similarities, such as six streaks which arise from the hexagonal cross section of the wires. The diffraction patterns along each wire (i.e., at different heights) are also different, demonstrating that their structure evolves along the wire for all of them. The diffraction patterns are more complex 

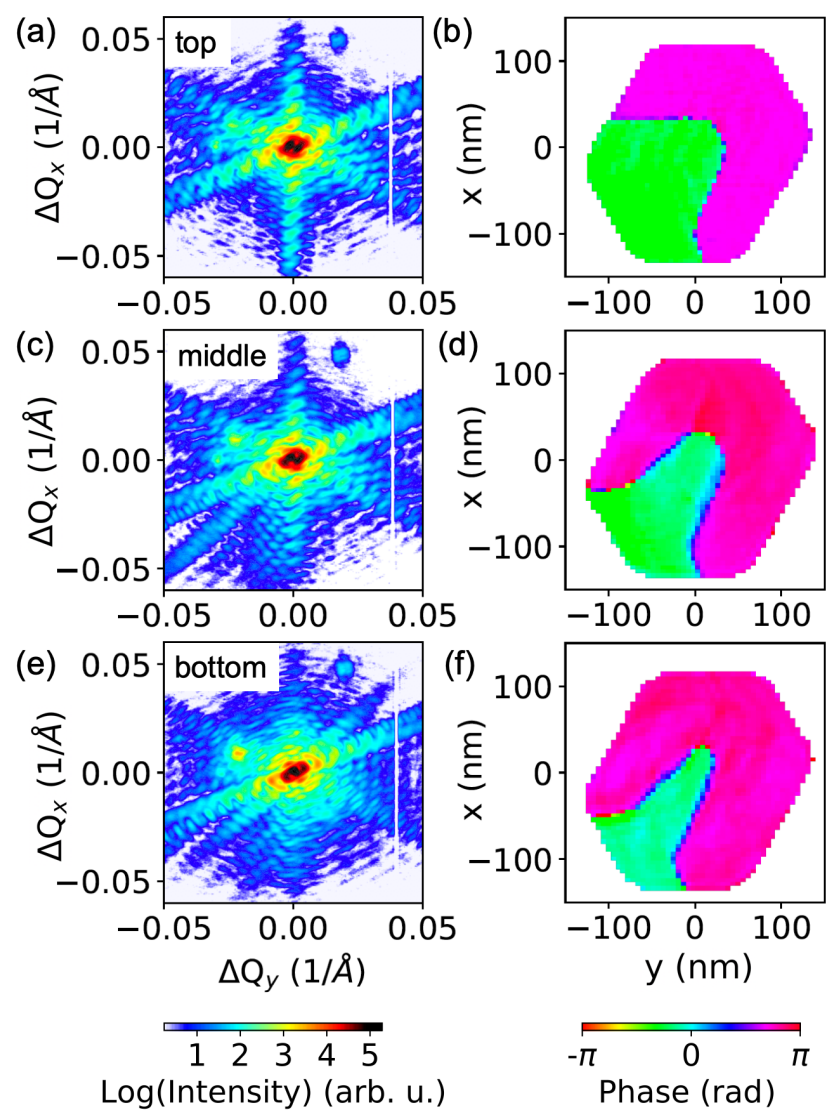

Figure 3: Coherent x-ray diffraction measurements of the $004 \mathrm{GaN}$ Bragg peak (left) and their associated real space reconstruction of the phase (right) at the top (a-b), center (c-d) and bottom (e-f) of the $3.6 \mu \mathrm{m}$ long wire $B$.

at the bottom of the wires, as expected, due to the threading dislocations nucleated at the interface between the GaN wire and the substrate that bend towards the sidewall free surface to minimise the free energy of the system. ${ }^{11,23}$ Each diffraction pattern was reconstructed independently with phase retrieval algorithms to reveal the internal structure of the wires.

Phase retrieval was carried out on the diffraction data using the PyNX package, ${ }^{24}$ imposing at each iteration that the calculated Fourier intensity of the guessed object, $\tilde{\Omega}(\vec{r})$, agrees with the measured data, $I^{\text {meas }}$ (see the Methods and Experimental Section). To select pertinent results, the Poisson Log-Likelihood (LLK) ${ }^{25}$ is used as a metric to quantify the quality of phasing. LLK is defined as:

$$
L L K=-\frac{1}{N} \sum_{i} \log \left[\frac{\left(I_{i}^{c a l c}\right)^{I_{i}^{\text {meas }}}}{I_{i}^{\text {meas }} !} e^{-I_{i}^{\text {calc }}}\right]
$$


(a)
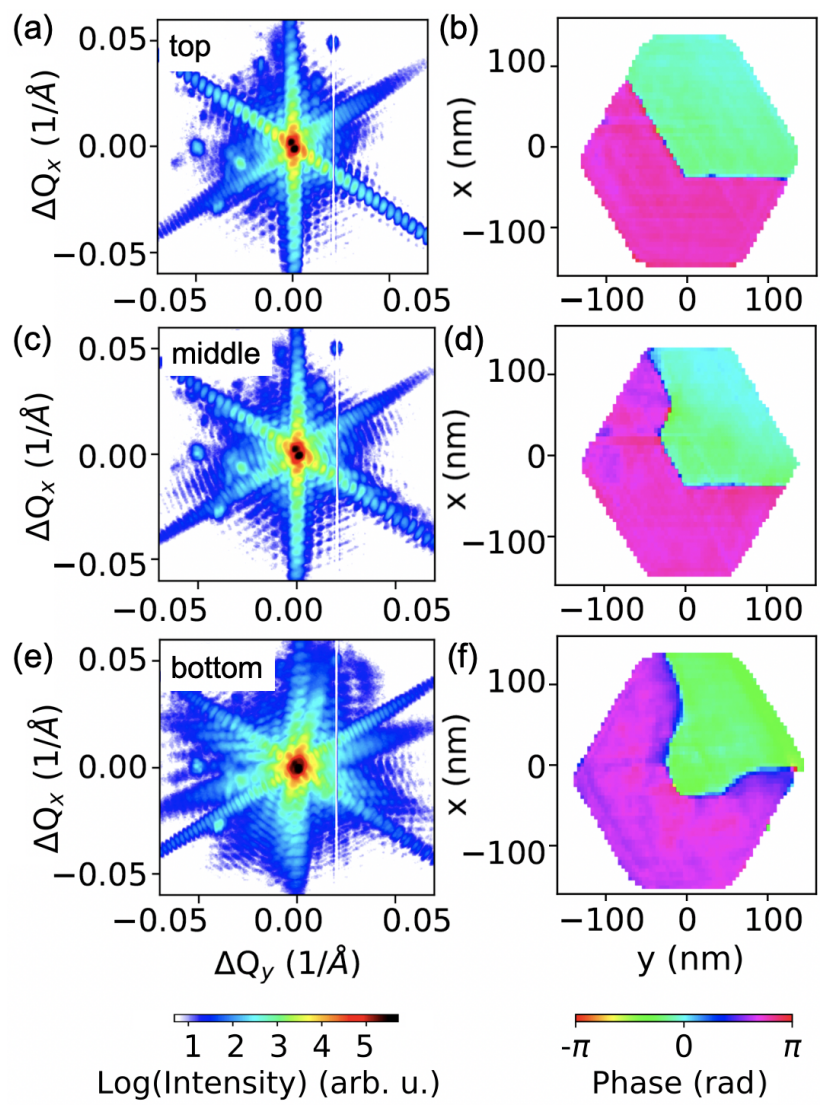

Figure 4: Coherent x-ray diffraction measurements of the $004 \mathrm{GaN}$ Bragg peak (left) and their associated real space reconstruction of the phase (right) at the top (a-b), center (c-d) and bottom (e-f) of the $4.3 \mu \mathrm{m}$ long wire $C$.

where $N$ is the total number of pixels in the diffracted data, $I_{i}^{\text {meas }}$ and $I_{i}^{\text {calc }}$ denote the measured and calculated intensity at pixel $i$. LLK reflects the similarity of calculated and observed intensities. Reconstructed objects with lower LLKs have more homogeneous phases and sharper boundaries between the inversion domains. The true object $\tilde{\Omega}(\vec{r})$ as well as its twin-image $\tilde{\Omega}^{*}(-\vec{r})$, also called enantiomorph, are parts of the reconstructed solutions, since they have the same intensity in reciprocal space. It was necessary to recognise the true object from the twin-image to obtain the correct phase shift between the inversion domains, as the phase of the twin-object is opposite to the correct phase. This is done by considering the effect of the optical path length on the phase distribution (see Supporting Information S2).

Figures 2, 3 and 4 (b, d and f) display the best reconstructed images of the wires at different heights. Two domains of different constant phase values (colored in pink and green) are observed. 
They correspond to a stepped phase contrast without a long-range strain field. The domains are reconstructed with a pixel size of 6 ( $x$-direction) $\times 8.5$ ( $y$-direction) $\mathrm{nm}^{2}$ for wire $A$, a pixel size of $4.9(x) \times 5.6(y) \mathrm{nm}^{2}$ for wire $B$ and a pixel size of $5.1(x) \times 4.2(y) \mathrm{nm}^{2}$ for wire $C$. They correspond to domains of different polarities, either Ga $(+c)$ or $\mathrm{N}(-c)$ terminated domains, separated by inversion domain boundaries as reported in Ref. ${ }^{16}$ The in-plane spatial resolution of the reconstructed images has been evaluated to $9 \mathrm{~nm}$ using two methods: the Phase Retrieval Transfer Function (PRTF) ${ }^{26,27}$ and the Fourier Shell Correlation (FSC) ${ }^{28-30}$ (see Supporting Information S3).
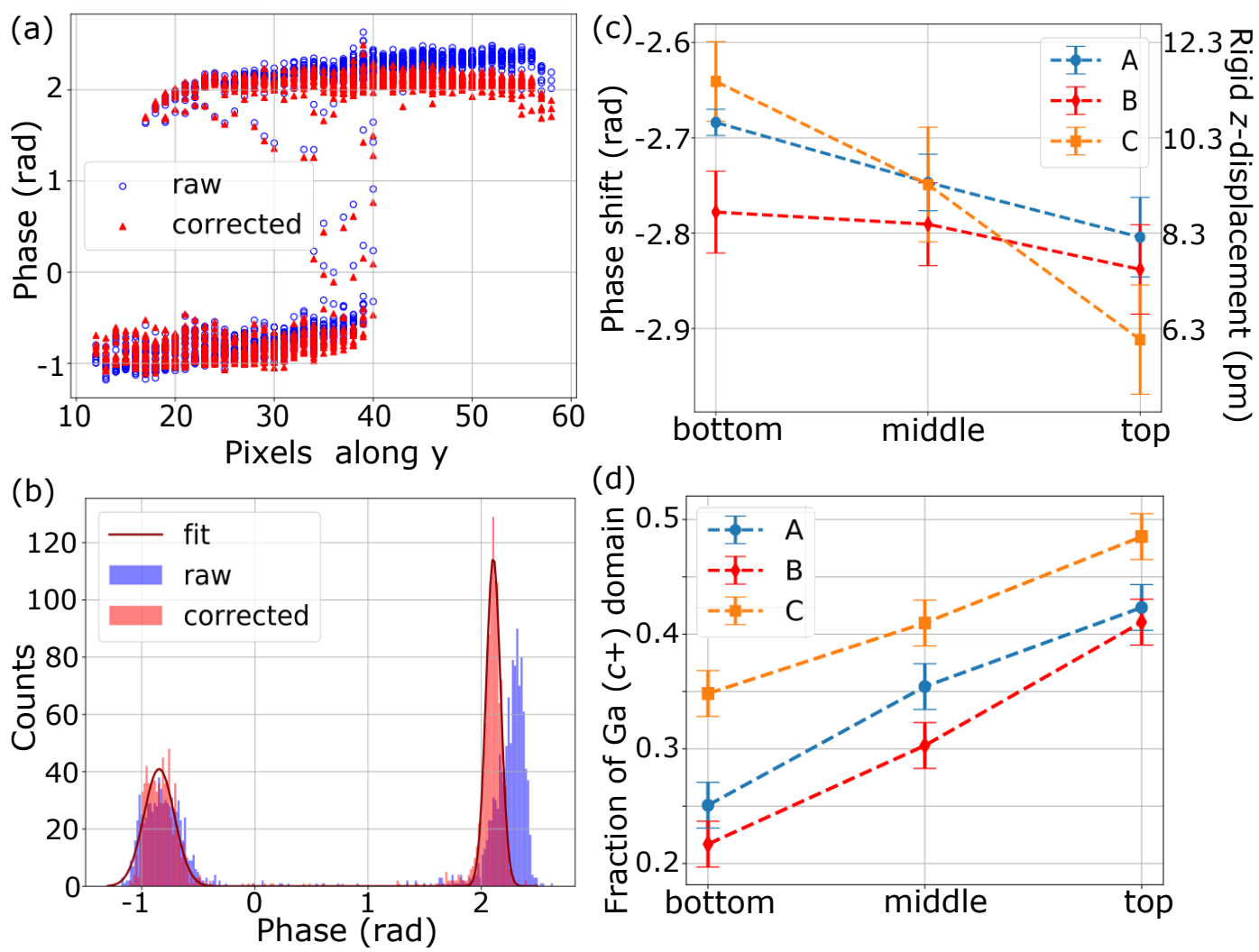

(d)

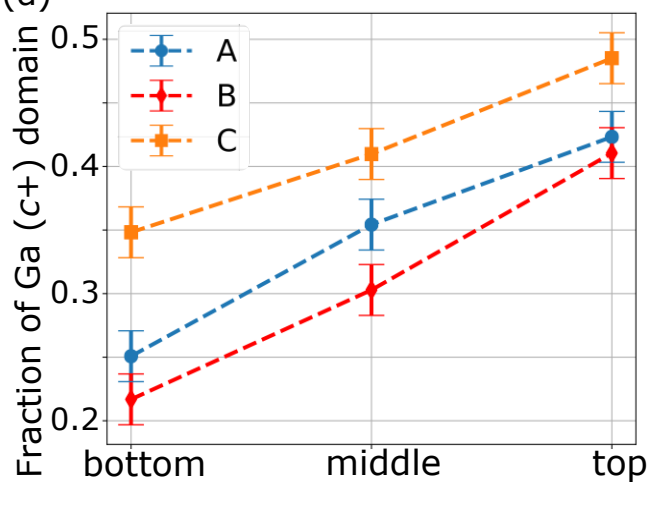

Figure 5: (a) Raw and corrected values of the phase distribution along the $y$-axis of the reconstructed top cross-section of wire $B$. (b) Histograms of the phase of the raw and corrected (after optical path and phase ramp corrections) of the reconstructed top cross-section of wire $B$. Gaussian fit of the corrected phase histogram. (c) Evolution of the phase shift and corresponding rigid outof-plane displacement between inversion domains at the bottom, middle and top of the different wires. (d) Evolution of the fraction of $\mathrm{Ga}(c+)$ terminated domain along the different wires.

Figure 5(a) displays the raw and corrected values of the phase distribution along the $y$-axis of the reconstructed top cross-section of wire $B$ (shown in Figure 3(b)). To get a correct phase-shift, 
the retrieved phases are corrected for the optical path (i.e. from x-ray refraction and absorption). The phase-ramp, which corresponds to an uncontrolled degree of freedom in the reconstruction, ${ }^{31}$ is removed. ${ }^{32,33}$ Histograms of the raw and corrected phase distributions are displayed in Figure 5(b). Two main peaks are observed, corresponding to the two different domains (either $+c$ or $-c$ ) of the GaN wire. These two peaks were fitted by Gaussian functions to get the phase shift between the inversion domains. The average phase shift and its standard deviation (see Figure 5(c)) were estimated from 1200 different reconstructions (i.e., 1200 different initial random phases), all of them showing a low value of their log-likelihood (LLK) metric. ${ }^{25}$ As shown in Figure 5(c), the retrieved phase shift for inversion domains is slightly different depending on the height along the wires. For instance, the phase shift equals to $-2.68 \pm 0.02 \mathrm{rad},-2.75 \pm 0.03 \mathrm{rad}$ and $-2.80 \pm 0.04$ rad at the bottom, center and top parts of the wire $A$. A slight absolute increase of the phase-shift is thus observed from the bottom to the top for all the wires. Note that the bottom part value is probably still affected by the interface between the GaN wire and the sapphire substrate containing dislocations and that this point applies to the three wires. The medium position measurements exhibit a small distribution of the phase shift $(-2.76 \pm 0.02 \mathrm{rad})$ and the comparison of the upper values do not show a significant change between wire $A$ (constant silane flow) and wire $B$ (varied silane flow), whereas wire $C$ (also with silane flow variation) has a significantly lower value. These observations show that the Si-content in the wires does not play a major role on the $c$-axis IDB displacement.

To analyse these phase differences, the structure factor of Ga and N-terminated GaN domains has to be considered first. Supposing that the Ga atoms are positioned at $(0,0,0)$ and $\left(\frac{1}{3}, \frac{2}{3}, \frac{1}{2}\right)$ and $\mathrm{N}$ atoms at $(0,0, z)$ and $\left(\frac{1}{3}, \frac{2}{3}, \frac{1}{2}+z\right)$ in the unit cell of the Ga-polar $(+c)$ domains, the structure factors of the $+c$ and $-c$ domains follow, for a given $\boldsymbol{h} \boldsymbol{k l}$ reflection:

$$
\begin{aligned}
\tilde{F}_{+c}(h k l)= & \tilde{f}_{N}(h k l) \cdot\left\{\exp [-2 \pi i l z]+\exp \left[-2 \pi i\left(\frac{h}{3}+\frac{2 k}{3}+\frac{l+2 z}{2}\right)\right]\right\} \\
& +\tilde{f}_{G a}(h k l) \cdot\left\{1+\exp \left[-2 \pi i\left(\frac{h}{3}+\frac{2 k}{3}+\frac{l}{2}\right)\right]\right\}
\end{aligned}
$$




$$
\begin{aligned}
\tilde{F}_{-c}(h k l)= & \tilde{f}_{G a}(h k l) \cdot\left\{\exp [-2 \pi i l z]+\exp \left[-2 \pi i\left(\frac{h}{3}+\frac{2 k}{3}+\frac{l+2 z}{2}\right)\right]\right\} \\
& +\tilde{f}_{N}(h k l) \cdot\left\{1+\exp \left[-2 \pi i\left(\frac{h}{3}+\frac{2 k}{3}+\frac{l}{2}\right)\right]\right\}
\end{aligned}
$$

where $\tilde{f}_{N}(h k l)$ and $\tilde{f}_{G a}(h k l)$ are the (complex) atomic scattering factors for $\mathrm{N}$ and Ga atoms, respectively. $z\left(=0.377\right.$ in $c$-lattice unit $\left.{ }^{34}\right)$ is the distance between two basal planes with atoms of different types in the wurtzite GaN unit cell. The diffracted amplitude of the wire with two domains $(+c$ and $-c)$ can be written as:

$$
\begin{aligned}
\tilde{A}(\vec{Q}) \propto \quad & F T\left[\Omega_{+c} \cdot \tilde{F}_{+c}(h k l) \cdot \exp \left(-i \vec{Q}_{0} \cdot \vec{u}_{+c}\right)\right. \\
& \left.+\Omega_{-c} \cdot \tilde{F}_{-c}(h k l) \cdot \exp \left(-i \vec{Q}_{0} \cdot \vec{u}_{-c}\right)\right]
\end{aligned}
$$

where $\Omega_{+c}\left(\Omega_{-c}\right)=1$ inside the $+c(-c)$ domain and 0 outside, $\vec{Q}_{0}$ is the reference scattering vector in reciprocal space (corresponding to the measured $\boldsymbol{h} \boldsymbol{k} \boldsymbol{l}$ reflection). $\vec{u}_{+c}$ and $\vec{u}_{-c}$ are the rigid displacements of each domain. FT stands for Fourier transform. The retrieved complex-valued object then verifies:

$$
\begin{aligned}
\tilde{\Omega}(\vec{r})= & \Omega_{+c} \cdot \tilde{F}_{+c}(h k l) \cdot \exp \left(-i \vec{Q}_{0} \cdot \vec{u}_{+c}\right) \\
& +\Omega_{-c} \cdot \tilde{F}_{-c}(h k l) \cdot \exp \left(-i \vec{Q}_{0} \cdot \vec{u}_{-c}\right) .
\end{aligned}
$$

The difference of phase between the two domains, $\Delta \Phi$, originates from the difference of phase of the structure factor between $\mathrm{Ga}-(+c)$ and $\mathrm{N}-(-c)$ polarity domains and from their relative rigid displacements:

$$
\Delta \Phi_{-c \rightarrow+c}=\Phi_{\tilde{F}_{-c}}-\Phi_{\tilde{F}_{+c}}+\vec{Q}_{0} \cdot\left(\vec{u}_{+c}-\vec{u}_{-c}\right)
$$

For the $\mathbf{0 0 4} \mathrm{GaN}$ reflection and the $\mathrm{Ga}$ and $\mathrm{N}$ analytical atomic scattering factors taken from the work of Waasmaier and Kirfel, ${ }^{35} \Phi_{\tilde{F}_{-c}}-\Phi_{\tilde{F}_{+c}}=3.08$ (or -3.2) rad. The measured phaseshift of the wire $A$ varies from $-2.68 \pm 0.02 \mathrm{rad}$ (bottom) to $-2.80 \pm 0.04 \mathrm{rad}$ (top). These values are different from $\left(\Phi_{\tilde{F}_{-c}}-\Phi_{\tilde{F}_{+c}}\right)$; this demonstrates the necessity to add a rigid displacement 
along the $z$-direction between the $+c$ and $-c$ domains varying from $(c / 2+10.8) \pm 0.3 \mathrm{pm}$ (bottom) to $(c / 2+8.3) \pm 0.9 \mathrm{pm}$ (top). The phase-shift for wire $B$ varies from $-2.78 \pm 0.04 \mathrm{rad}$ (bottom) to $-2.84 \pm 0.05 \mathrm{rad}$ (top), i.e. a variation of the displacement from $(c / 2+8.8) \pm 0.5 \mathrm{pm}$ (bottom) to $(c / 2+7.5) \pm 0.5 \mathrm{pm}$ (top). Wire $C$ shows the largest variation: from $-2.64 \pm 0.04 \mathrm{rad}$ (bottom) to $-2.91 \pm 0.06 \mathrm{rad}$ (top), i.e. from $(c / 2+11.5) \pm 1.0 \mathrm{pm}$ (bottom) to $(c / 2+6) \pm 1 \mathrm{pm}$ (top). The experimental shift of the two domains along the hexagonal $c$-axis (varying from $c / 2+6 \mathrm{pm}$ at minimum to $c / 2+11.5 \mathrm{pm}$ at maximum) is in agreement with previous BCDI results ${ }^{16}$ and with the atomistic $a b$ initio calculations of Lançon et al. ${ }^{36}$ adding a $8 \mathrm{pm} c$-displacement to the usual $c / 2$ value shift of the initial Northrup et al. IDB* model. ${ }^{37}$ This model is one of the four structures of $\{1 \overline{1} .0\}$ IDB reported in the literature. ${ }^{37,38}$ From these values consistent with Ref. ${ }^{16}$ where multiple reflections have been probed, we can assert that for all wires, the domains in pink in Figures 2, 3 and 4 are $-c$ (N-terminated) and the ones in green are $+c$ (Ga-terminated). Note that results of only three wires are shown here but thirty wires were measured. They were all located at the edges of the substrate to increase the probability of IDB defects inside the wires. All the measured wires show inversion domains. The size of the inversion domains fluctuates but inversion domains of IDB*-type were always observed.

It has been extensively shown in literature that polarity inversion is generally occurring at the interface between GaN and sapphire both in planar and (nano-)wire growths. ${ }^{5,39-41}$ They nucleate at the first stage of the growth on different types of defects such as surface steps, and more generally on morphological and chemical heterogeneities, and they can interact with other defects, e.g. threading dislocations and stacking faults. ${ }^{42}$ The polarity mixing control poses a key challenge for the growth techniques in particular for molecular beam epitaxy ${ }^{43}$ and MOVPE. ${ }^{44}$ Within the MOVPE growth conditions of this study, the annealing of sapphire under ammonia promotes an AlN-terminated surface and consequently a majority of N-polar wires at the wafer scale. This tendency is observed at the bottom of the three wires with $\mathrm{N}$-polar domains (pink color) having the largest size. Figure 5(d) displays the evolution of the fraction of the $\mathrm{Ga}(+c)$ terminated domain along the different wires. At larger height and for these quite short wires, N-polar domains shrink 
in favor of the Ga-domain (note that this effect has been already observed by the optical signature of the two types of domains in Ref. ${ }^{8}$ ), but the complete wire keeps roughly the same cross-section. The expansion of the $+c$ or Ga polar domain for all wires is in agreement with the usual tendency of this type of wire growth. This can be explained by the different speed of vertical and lateral growths of Ga polar domains compared to $\mathrm{N}$ polar domains. ${ }^{5,39}$ This expansion (increase of dimension by a factor of $\sim 1.7$ from bottom to top of the wires) indicates that the IDB* propagation is not straight in the prismatic $m$-plane. Such IDB* can interact with stacking faults in the basal plane ${ }^{40,42,45}$ to induce an in-plane shift of the propagation along the $c$-axis, and create also macrofacetting by dislocation climbing, as mentioned by Coulon et al. ${ }^{46,47}$ in $\{10 \overline{1} 4\}$ planes for GaN and $\{10 \overline{1} 3\}$ for $\mathrm{ZnO} .{ }^{48}$ Our observations also show that the IDBs* stabilise in the $\{1 \overline{1} 00\}$ planes, as the IDBs* at the top of the wires are all parallel to the $m$-planes (see Figures 2,3 and 4(b)), while it is not the case at the bottom of the wires (see Figures 2, 3 and 4(f)). This implies that the IDBs* adopt different configurations before stabilising into $m$-planes. It is very likely that the fast vertical growth promotes their expansion in the $\{10 \overline{1} 0\}$ planes, and that the difference of rigid displacement between the middle and top of the wires are related to the stabilisation of the IDBs onto $\{1 \overline{1} 00\}$ planes. Interestingly, we do not observe long-range strain at the interface of the boundaries, as only a stepped-phase contrast was observed at the IDBs* for all the different positions along the wires. We do see an evolution of the relative rigid displacement between domains $(\sim 5$ pm, at maximum) along the wires. As the evolution of this rigid displacement does not strongly differ with or without silane, the Si-content in the wires does not play a major role on the $c$-axis IDB* displacement. The modification of configuration of the IDBs* along the wires may explain the slight change of the rigid displacement between domains. The variation along the wire length is difficult to relate to the $\mathrm{Si}$ content, indeed the average value is very close to the $a b$ initio calculation performed without $\mathrm{Si}$ and first-principle calculations ${ }^{36}$ showed that silicon incorporation does not affect the lattice constant of the GaN crystal. ${ }^{49}$ In 2D material, the increase of stress has been proposed to be related to the presence of tensile stress due to crystallite coalescence. ${ }^{50}$ Within our materials, the non-straight IDB* configuration may play a similar role and create small local stress 
evolving also as a function of polarity fraction, i.e. continuously as a function of length.

\section{Conclusions}

We have studied the inversion domain boundary distribution in cross-sections of self-catalysed and silane-assisted MOVPE wires grown along their growth axis. This nm-resolved structural analysis provide understanding on their evolution along the wire length. The IDBs were found to move along the growth axis, promoting Ga-terminated domains and stabilising into $\{1 \overline{1} 00\}$ (i.e. $m$-planes) over N-terminated domains. The $c$-axis distance between domains was found to vary from $c / 2+6 \mathrm{pm}$ at minimum to $c / 2+11.5 \mathrm{pm}$ at maximum from top to bottom. The resolution on the displacement along the $c$-axis (in the order of $1 \mathrm{pm}$ ) is an invaluable output of Bragg coherent diffraction imaging. The observed picometer-variation of the rigid displacement between the domains is related to the modification of the configuration of the IDBs* along the wires (influence of interfacial defects at the bottom of the wires, macrofacetting, etc.). Silicon doping has no noticeable effect on the $c$-axis IDB* displacement. The BCDI technique offers a very precise inner view of the microstructure of small crystals in the presence of interacting defects. The technique can be extended to materials other than GaN. It can be also applied in a straightforward manner to materials under a complex environment or operando as found in microelectronics and optoelectronics devices.

\section{Methods/Experimental}

Growth Wires are grown by metal organic vapor phase epitaxy (MOVPE) on $c$-plane sapphire substrates in a 3 x 2" closed-coupled shower-head reactor using ammonia $\left(\mathrm{NH}_{3}\right)$ and trimethylgallium (TMG) precursor. We used a self-catalysed and silane-assisted method that can be summarised as followed (see further details in Ref. ${ }^{51}$ ). The substrate is first cleaned under $\mathrm{H}_{2}$ at high temperature and annealed under $\mathrm{NH}_{3}$ to promote the formation of an $\mathrm{Al}(\mathrm{O}) \mathrm{N}$ layer before the deposition of a thin $\operatorname{SiN}_{x}$ layer ( $\sim 2 \mathrm{~nm}$ thick), which plays the role of a selective growth layer with respect to 
GaN. ${ }^{52}$ A second $\mathrm{NH}_{3}$ annealing stabilises the surface stoichiometry and induces the formation of composition fluctuations or thinner areas that act as nucleation sites for the GaN epitaxial growth on sapphire. The GaN polarity of the wires are directly determined by the atomic stacking of the $\mathrm{Al}(\mathrm{O}) \mathrm{N}$ layer ${ }^{53}$ and their shapes are also related to the morphology of the initial seed. To promote the vertical growth, we use a silane addition of $203 \mathrm{nmol} / \mathrm{min}$ diluted in a carrier gas mainly $\mathrm{N}_{2}$ and a small V/III molar ratio $\left(\left[\mathrm{NH}_{3}\right] /[\mathrm{TMG}]=49.5\right)$, the volume flow of $\mathrm{NH}_{3}$ and $\mathrm{TMG}$ being 150 and $60 \mathrm{sccm}$, respectively in a total nitrogen flow of $4000 \mathrm{sccm}$. The growth is performed at 1050 ${ }^{\circ} \mathrm{C}$ and 800 mbar. Adjusting these growth conditions for BCDI allows to achieve a low density ( $\sim 10^{6} \mathrm{~cm}^{-2}$ ) of wires with a length ranging from 3 to $5 \mu \mathrm{m}$ and an average diameter of $600 \mathrm{~nm}$, as illustrated by the scanning electron microscope image (see Figure 1). Moreover, we selected preferentially areas at the edges of the substrate to have access to an even lower density and to illuminate only single wires under the beam. The edges also benefit from an increased probability of inversion domain boundary (IDB) defects inside wires. Silane addition results in a heavily $n^{++}$ doped GaN wire (the concentration of of electrons in the donor energy state is $\mathrm{N}_{d} \sim 10^{20} \mathrm{~cm}^{-3}$ ) 51 that is used to facilitate current injection in devices. ${ }^{8,12,13}$ In this case, a thin $\mathrm{SiN}_{x}$ passivation layer is formed on the $m$-plane $\mathrm{GaN}$ wire sidewalls that prevents any lateral growth and also strongly favors the vertical (longitudinal) growth. When the silane is switched off, the longitudinal growth continues consuming the remaining silane concentration in the reactor chamber. It also provides a residual $n^{+}$doped GaN part $\left(\mathrm{N}_{d} \sim 10^{19} \mathrm{~cm}^{-3}\right)$ at the top of the wire.

Bragg coherent diffraction imaging Experiments were performed at the ID01 beamline of the European Synchrotron Facility (ESRF). A coherent portion of the monochromatic $(9 \mathrm{keV})$ beam was selected with high precision slits. The coherent beam was then focused using a Fresnel Zone Plate (diameter of $300 \mu \mathrm{m}$ ), to illuminate a single GaN wire. Diffraction was measured by a twodimensional detector $(516 \times 516$ pixels of $55 \mu \mathrm{m}$ placed at $1.307 \mathrm{~m}$ (for wire $A$ ) or at $0.676 \mathrm{~m}$ (for wires $B$ and $C$ ) from the sample). 3D data sets were recorded by rocking the sample over a range of $1.28^{\circ}$ (for wire $A$ ) or $1.5^{\circ}$ (for wires $B$ and $C$ ) by steps of $0.005^{\circ}$.

Phase retrieval algorithm The intensity recorded on the 2D detector is masked to select only 
the diffraction pattern of the investigated wire. The masked intensity is let free during phase retrieval. Starting with random objects, 1200 iterations of Relaxed Averaged Alternating Reflections $\left(\mathrm{RAAR}^{54}\right)$ and 300 iterations of Error-Reduction $\left(\mathrm{ER}^{55,56}\right)$ algorithms were applied with shrinkwrap $^{57}$ algorithm (every 20 iterations) in each phasing process. After repeating several times, a support was built from the best reconstructed object. With this support as a starting support, it converges faster to the expected direct-space object. Afterwards, the support was fixed and it converges fairly well with 600 iterations of Hybrid Input Output $\left(\mathrm{HIO}^{58}\right), 600$ iterations of RAAR and 300 iterations of ER. Although the support is fixed, the starting phase before each phasing process is totally random, implying that the generated phases are slightly different with each other. This is on account of noise caused by the detector. The removal of the phase-ramp of the reconstructed data is achieved by performing linear-regression on the two domains one by one and by calculating the weighted mean slope.

\section{Acknowledgement}

The authors are grateful to ESRF Synchrotron for allocating beamtime. The measurement was performed at the ID01-ESRF beamline. We thank ID01 beamline staff for excellent support during the experiment. This work has been funded by the French National Research Agency through the project ANR-11-BS10-01401 MecaniX. This project has also received funding from the European Research Council (ERC) under the European Union's Horizon 2020 research and innovation programme (grant agreement No. 818823).

\section{Supporting Information Available}

The Supporting Information is available free of charge on the ACS Publications website at DOI: Additional information on the height of the wires, the recognition of the twin-image and the spatial resolution $(\mathrm{PDF})$ 


\section{References}

(1) Nakamura, S.; Mukai, T.; Senoh, M. Candela-Class High-Brightness InGaN/AlGaN DoubleHeterostructure Blue-Light-Emitting Diodes. Appl. Phys. Lett. 1994, 64, 1687-1689.

(2) Jung, H. S.; Hong, Y. J.; Li, Y.; Cho, J.; Kim, Y.-J.; Yi, G.-C. Photocatalysis Using GaN Nanowires. ACS Nano 2008, 2, 637-642.

(3) Pearton, S. J.; Ren, F. GaN Electronics. Adv. Mater. 2000, 12, 1571-1580.

(4) Lester, S.; Ponce, F.; Craford, M.; Steigerwald, D. High Dislocation Densities in High Efficiency GaN-Based Light-Emitting Diodes. Appl. Phys. Lett. 1995, 66, 1249-1251.

(5) Romano, L. T.; Northrup, J. E.; O’Keefe, M. A. Inversion Domains in GaN Grown on Sapphire. Appl. Phys. Lett. 1996, 69, 2394-2396.

(6) Chen, X. J.; Perillat-Merceroz, G.; Sam-Giao, D.; Durand, C.; Eymery, J. Homoepitaxial Growth of Catalyst-Free GaN Wires on N-Polar Substrates. Appl. Phys. Lett. 2010, 97, 151909.

(7) Li, S. F.; Fuendling, S.; Wang, X.; Merzsch, S.; Al-Suleiman, M. A. M.; Wei, J. D.; Wehmann, H.-H.; Waag, A.; Bergbauer, W.; Strassburg, M. Polarity and Its Influence on Growth Mechanism during MOVPE Growth of GaN Sub-micrometer Rods. Cryst. Growth Des. 2011, 11, 1573-1577.

(8) Salomon, D.; Messanvi, A.; Eymery, J.; Martínez-Criado, G. Silane-Induced N-Polarity in Wires Probed by a Synchrotron Nanobeam. Nano Lett. 2017, 17, 946-952.

(9) Stutzmann, M.; Ambacher, O.; Eickhoff, M.; Karrer, U.; Lima Pimenta, A.; Neuberger, R.; Schalwig, J.; Dimitrov, R.; Schuck, P.; Grober, R. Playing with Polarity. Phys. Status Solidi B 2001, 228, 505-512. 
(10) Ambacher, O.; Majewski, J.; Miskys, C.; Link, A.; Hermann, M.; Eickhoff, M.; Stutzmann, M.; Bernardini, F.; Fiorentini, V.; Tilak, V.; Schaff, B.; Eastman, L. F. Pyroelectric Properties of $\mathrm{Al}(\mathrm{In}) \mathrm{GaN} / \mathrm{GaN}$ Hetero- and Quantum Well Structures. J. Phys.: Condens. Matter 2002, 14, 3399-3434.

(11) Kishino, K.; Ishizawa, S. Selective-Area Growth of GaN Nanocolumns on Si(111) Substrates for Application to Nanocolumn Emitters with Systematic Analysis of Dislocation Filtering Effect of Nanocolumns. Nanotechnol. 2015, 26, 225602.

(12) Dai, X.; Messanvi, A.; Zhang, H.; Durand, C.; Eymery, J.; Bougerol, C.; Julien, F. H.; Tchernycheva, M. Flexible Light-Emitting Diodes Based on Vertical Nitride Nanowires. Nano Lett. 2015, 15, 6958-6964.

(13) El Kacimi, A.; Pauliac-Vaujour, E.; Eymery, J. Flexible Capacitive Piezoelectric Sensor with Vertically Aligned Ultralong GaN Wires. ACS Appl. Mater. Interfaces 2018, 10, 4794-4800.

(14) Liu, F.; Collazo, R.; Mita, S.; Sitar, Z.; Pennycook, S. J.; Duscher, G. Direct Observation of Inversion Domain Boundaries of GaN on c-Sapphire at Sub-ångstrom Resolution. Adv. Mater. 2008, 20, 2162-2165.

(15) Robinson, I.; Harder, R. Coherent X-Ray Diffraction Imaging of Strain at the Nanoscale. Nat. Mater. 2009, 8, 291-298.

(16) Labat, S.; Richard, M.-I.; Dupraz, M.; Gailhanou, M.; Beutier, G.; Verdier, M.; Mastropietro, F.; Cornelius, T. W.; Schülli, T. U.; Eymery, J.; Thomas, O. Inversion Domain Boundaries in GaN Wires Revealed by Coherent Bragg Imaging. ACS Nano 2015, 9, 9210-9216.

(17) Ulvestad, A.; Singer, A.; Clark, J. N.; Cho, H. M.; Kim, J. W.; Harder, R.; Maser, J.; Meng, Y. S.; Shpyrko, O. G. Topological Defect Dynamics in Operando Battery Nanoparticles. Science 2015, 348, 1344-1347. 
(18) Hofmann, F.; Tarleton, E.; Harder, R. J.; Phillips, N. W.; Ma, P.-W.; Clark, J. N.; Robinson, I. K.; Abbey, B.; Liu, W.; Beck, C. E. 3D Lattice Distortions and Defect Structures in Ion-Implanted Nano-Crystals. Sci. Rep. 2017, 7, 45993.

(19) Favre-Nicolin, V.; Mastropietro, F.; Eymery, J.; Camacho, D.; Niquet, Y. M.; Borg, B. M.; Messing, M. E.; Wernersson, L.-E.; Algra, R. E.; Bakkers, E. P. a. M.; Metzger, T. H.; Harder, R.; Robinson, I. K. Analysis of Strain and Stacking Faults in Single Nanowires using Bragg Coherent Diffraction Imaging. New J. Phys. 2010, 12, 035013.

(20) Hill, M. O.; Calvo-Almazan, I.; Allain, M.; Holt, M. V.; Ulvestad, A; Treu, J.; Koblmüller, G.; Huang, C.; Huang, X.; Yan, H.; Nazaretski, E.; Chu, Y. S.; Stephenson, G. B.; Chamard, V.; Lauhon, L. J.; Hruszkewycz, S. O. Measuring Three-Dimensional Strain and Structural Defects in a Single InGaAs Nanowire Using Coherent X-ray Multi-Angle Bragg Projection Ptychography. Nano Lett. 2018, 18, 811-819.

(21) Davtyan, A.; Favre-Nicolin, V.; Lewis, R. B.; Küpers, H.; Geelhaar, L.; Kriegner, D.; Bahrami, D.; Al-Hassan, A.; Chahine, G.; Loffeld, O.; Pietsch, U. Coherent X-Ray Diffraction Imaging Meets Ptychography to Study Core-Shell-Shell Nanowires. MRS Adv. 2018, 3, $2317-2322$.

(22) Dupraz, M. Coherent X-Ray Diffraction Applied to Metal Physics. Ph.D. thesis, Grenoble Alpes University, Grenoble-France, 2015.

(23) Gibart, P.; Beaumont, B.; Vennéguès, P. In Nitride Semiconductors; Ruterana, P., Albrecht, M., Neugebauer, J., Eds.; Wiley-VCH Verlag GmbH \& Co. KGaA: Weinheim, FRG, 2006; pp 45-106.

(24) Mandula, O.; Elzo Aizarna, M.; Eymery, J.; Burghammer, M.; Favre-Nicolin, V. PyNX.Ptycho: a Computing Library for X-Ray Coherent Diffraction Imaging of Nanostructures. J. Appl. Crystallogr. 2016, 49, 1842-1848. 
(25) Thibault, P.; Guizar-Sicairos, M. Maximum-Likelihood Refinement for Coherent Diffractive Imaging. New J. Phys. 2012, 14, 063004.

(26) Chapman, H. N.; Barty, A.; Marchesini, S.; Noy, A.; Hau-Riege, S. P.; Cui, C.; Howells, M. R.; Rosen, R.; He, H.; Spence, J. C. H.; Weierstall, U.; Beetz, T.; Jacobsen, C.; Shapiro, D. High-Resolution Ab Initio Three-Dimensional X-Ray Diffraction Microscopy. J. Opt. Soc. Am. A 2006, 23, 1179-1200.

(27) Cherukara, M. J.; Cha, W.; Harder, R. J. Anisotropic Nano-Scale Resolution in 3D Bragg Coherent Diffraction Imaging. Appl. Phys. Lett. 2018, 113, 203101.

(28) Rosenthal, P. B.; Henderson, R. Optimal Determination of Particle Orientation, Absolute Hand, and Contrast Loss in Single-Particle Electron Cryomicroscopy. J. Mol. Biol. 2003, $333,721-745$.

(29) Van Heel, M.; Schatz, M. Fourier Shell Correlation Threshold Criteria. J. Struct. Biol. 2005, $151,250-262$.

(30) Holler, M.; Diaz, A.; Guizar-Sicairos, M.; Karvinen, P.; Färm, E.; Härkönen, E.; Ritala, M.; Menzel, A.; Raabe, J.; Bunk, O. X-Ray Ptychographic Computed Tomography at 16 nm Isotropic 3D Resolution. Sci. Rep. 2014, 4, 1-5.

(31) Stockmar, M.; Cloetens, P.; Zanette, I.; Enders, B.; Dierolf, M.; Pfeiffer, F.; Thibault, P. NearField Ptychography: Phase Retrieval for Inline Holography Using a Structured Illumination. Sci. Rep. 2013, 3, 1927.

(32) Carnis, J. BCDI Python package, version 0.0.5, https://github.com/carnisj/bcdi, 2019.

(33) Carnis, J.; Gao, L.; Labat, S.; Kim, Y. Y.; Hofmann, J. P.; Leake, S. J.; Schülli, T. U.; Hensen, E. J. M.; Thomas, O.; Richard, M.-I. Towards a Quantitative Determination of Strain 
in Bragg Coherent X-Ray Diffraction Imaging: Artefacts and Sign Convention in Reconstructions. Sci. Rep. 2019, 9, 1-13.

(34) Schulz, H.; Thiemann, K. H. Crystal Structure Refinement of AlN and GaN. Solid State Commun. 1977, 23, 815-819.

(35) Waasmaier, D.; Kirfel, A. New Analytical Scattering-Factor Functions for Free Atoms and Ions. Acta Crystallogr., Sect. A: Found. Crystallogr. 1995, 51, 416-431.

(36) Lançon, F.; Genovese, L.; Eymery, J. Towards Simulation at Picometer-Scale Resolution: Revisiting Inversion Domain Boundaries in GaN. Phys. Rev. B 2018, 98.

(37) Northrup, J.; Neugebauer, J.; Romano, L. Inversion Domain and Stacking Mismatch Boundaries in GaN. Phys. Rev. Lett. 1996, 77, 103-106.

(38) Moon, W. H.; Choi, C. H. Molecular-Dynamics Study of Inversion Domain Boundary in w-GaN. Phys. Lett. A 2006, 352, 538-542.

(39) Wu, X. H.; Fini, P.; Keller, S.; Tarsa, E. J.; Heying, B.; Mishra, U. K.; DenBaars, S. P.; Speck, J. S. Morphological and Structural Transitions in GaN Films Grown on Sapphire by Metal-Organic Chemical Vapor Deposition. Jpn. J. Appl. Phys. 1996, 35, L1648.

(40) Potin, V.; Nouet, G.; Ruterana, P. The 1010 Inversion Domains in GaN/Sapphire Layers: An Electron Microscopy Analysis of the Atomic Structure of the Boundaries. Philos. Mag. A 1999, 79, 2899-2919.

(41) Chen, X.; Hwang, J.; Perillat-Merceroz, G.; Landis, S.; Martin, B.; Dang, D. L. S.; Eymery, J.; Durand, C. Wafer-Scale Selective Area Growth of GaN Hexagonal Prismatic Nanostructures on c-Sapphire Substrate. J. Cryst. Growth 2011, 322, 15 - 22.

(42) Dimitrakopulos, G. P.; Komninou, P.; Kioseoglou, J.; Kehagias, T.; Sarigiannidou, E.; Georgakilas, A.; Nouet, G.; Karakostas, T. Structural Transition of Inversion Domain Boundaries Through Interactions with Stacking Faults in Epitaxial GaN. Phys. Rev. B 2001, 64, 245325. 
(43) Concordel, A.; Jacopin, G.; Gayral, B.; Garro, N.; Cros, A.; Rouvière, J.-L.; Daudin, B. Polarity Conversion of GaN Nanowires Grown by Plasma-Assisted Molecular Beam Epitaxy. Appl. Phys. Lett. 2019, 114, 172101.

(44) Wang, X.; Li, S.; Fündling, S.; Wei, J.; Erenburg, M.; Wehmann, H.-H.; Waag, A.; Bergbauer, W.; Strassburg, M.; Jahn, U.; Riechert, H. Polarity Control in 3D GaN Structures Grown by Selective Area MOVPE. Cryst. Growth Des. 2012, 12, 2552-2556.

(45) Corfdir, P.; Li, H.; Marquardt, O.; Gao, G.; Molas, M. R.; Zettler, J. K.; van Treeck, D.; Flissikowski, T.; Potemski, M.; Draxl, C.; Trampert, A.; Fernández-Garrido, S.; Grahn, H. T.; Brandt, O. Crystal-Phase Quantum Wires: One-Dimensional Heterostructures with Atomically Flat Interfaces. Nano Lett. 2018, 18, 247-254.

(46) Coulon, P. M.; Mexis, M.; Teisseire, M.; Jublot, M.; Vennéguès, P.; Leroux, M.; ZunigaPerez, J. Dual-polarity GaN Micropillars Grown by Metalorganic Vapour Phase Epitaxy: Cross-Correlation between Structural and Optical Properties. J. Appl. Phys. 2014, 115, 153504.

(47) Coulon, P.-M. Croissance et Caractérisation de Nanofils/Microfils de GaN. Ph.D. thesis, Nice-Sophia Antipolis University, Nice-France, 2014.

(48) Liu, Y. Z.; Yuan, H. T.; Zeng, Z. Q.; Du, X. L.; Han, X. D.; Xue, Q. K.; Zhang, Z. Inversion Domain Boundary in a ZnO Film. Philos. Mag. Lett. 2007, 87, 687-693.

(49) Romano, L. T.; Van de Walle, C. G.; Ager, J. W.; Götz, W.; Kern, R. S. Effect of Si Doping on Strain, Cracking, and Microstructure in GaN Thin Films Grown by Metalorganic Chemical Vapor Deposition. J. Appl. Phys. 2000, 87, 7745-7752.

(50) Nix, W. D.; Clemens, B. M. Crystallite Coalescence: A Mechanism for Intrinsic Tensile Stresses in Thin Films. J. Mater. Res. 1999, 14, 3467-3473. 
(51) Koester, R.; Hwang, J. S.; Durand, C.; Dang, D. L. S.; Eymery, J. Self-Assembled Growth of Catalyst-Free GaN Wires by Metal-Organic Vapour Phase Epitaxy. Nanotechnol. 2010, 21, 015602.

(52) Gibart, P. Metal Organic Vapour Phase Epitaxy of GaN and Lateral Overgrowth. Rep. Prog. Phys. 2004, 67, 667-715.

(53) Liu, F.; Collazo, R.; Mita, S.; Sitar, Z.; Duscher, G.; Pennycook, S. J. The Mechanism for Polarity Inversion of GaN via a Thin AlN Layer: Direct Experimental Evidence. Appl. Phys. Lett. 2007, 91, 203115.

(54) Luke, D. R. Relaxed Averaged Alternating Reflections for Diffraction Imaging. Inverse Probl. $\mathbf{2 0 0 4}, 21,37-50$.

(55) A Practical Algorithm for the Determination of the Phase from Image and Diffraction Plane Pictures.

(56) Fienup, J. Phase Retrieval Algorithms - a Comparison. Appl. Opt. 1982, 21, 2758-2769.

(57) Marchesini, S.; He, H.; Chapman, H. N.; Hau-Riege, S. P.; Noy, A.; Howells, M. R.; Weierstall, U.; Spence, J. C. H. X-Ray Image Reconstruction from a Diffraction Pattern Alone. Phys. Rev. B 2003, 68, 140101.

(58) Fienup, J. R.; Wackerman, C. C. Phase-Retrieval Stagnation Problems and Solutions. J. Opt. Soc. Am. A 1986, 3, 1897-1907.

\section{Graphical TOC Entry}




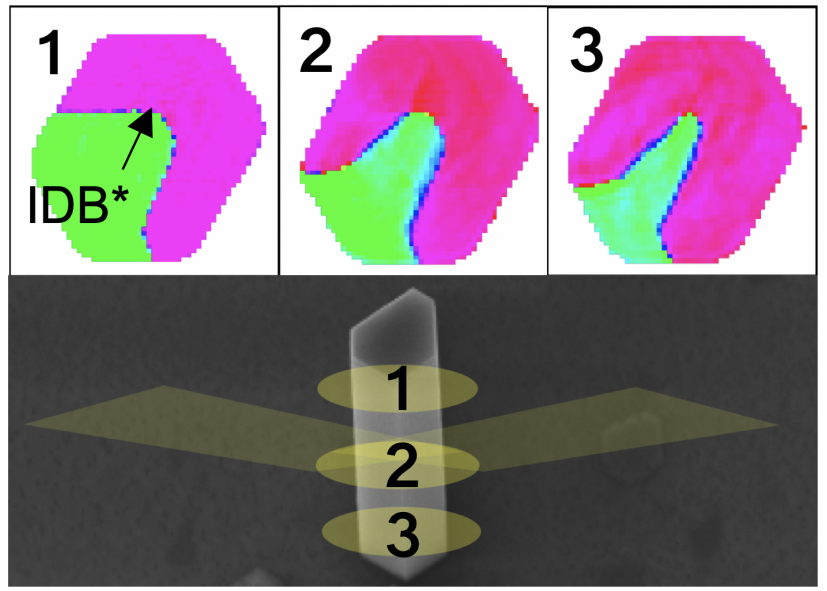

